\title{
Influence of Thermal Aging Phenomena on Thermoelectric Properties of Al-Substituted $\mathrm{ZnO}$
}

\author{
NINA VOGEL-SCHÄUBLE, ${ }^{1,3}$ RAPHAËL DUJARDIN ${ }^{1}$ \\ ANKE WEIDENKAFF, ${ }^{1,2}$ and MYRIAM H. AGUIRRE ${ }^{1,4}$ \\ 1.-Laboratory of Solid State Chemistry and Catalysis, Empa, Swiss Federal Laboratories \\ for Materials Science and Technology, Ueberlandstrasse 129, 8600 Duebendorf, Switzerland. \\ 2.—e-mail: anke.weidenkaff@empa.ch.3.—nina.vogel@empa.ch.4._myriam.aguirre@empa.ch
}

The thermoelectric properties and stability of $\mathrm{Al}$-substituted $\mathrm{ZnO}$ as a potential high-temperature $n$-type material were studied in heating-cooling cycles. $\mathrm{Zn}_{1-x} \mathrm{Al}_{x} \mathrm{O}(x=0.02,0.06)$ was prepared by soft chemistry and solidstate reaction synthesis methods. Cycling during the thermoelectric measurement leads to an increase of the electrical resistivity and Seebeck coefficient values. The reason for this aging phenomenon can be assigned to a change in composition due to oxygen uptake along with modification in the defect concentrations. The aging is enhanced if the cycling is performed in oxygen. $Z T$ value of 0.21 is reached at $1275 \mathrm{~K}$ for samples with $2 \% \mathrm{Al}$ substitution made by soft chemistry synthesis.

Key words: Thermoelectric properties, $\mathrm{Al}$-substituted $\mathrm{ZnO}$, soft chemistry, thermal cycling

\section{INTRODUCTION}

Thermoelectricity (TE) enables direct transformation of heat into electricity. The heat source can be, among others, from sunlight, geothermal, or excess heat from combustion processes. In TE modules, there is no need for mechanical parts that cause noise and abrasion, and there are no emissions. Suitable TE materials are those with figure of merit $Z T=S^{2} T / \rho \kappa>0.1$ (with temperature $T$, Seebeck coefficient $S$, electrical resistivity $\rho$, and thermal conductivity $\kappa$ ). State-of-the-art $\mathrm{Bi}_{2} \mathrm{Te}_{3}$-based materials display good TE properties. $Z$ value of $2.9 \times 10^{-3} \mathrm{~K}^{-1}$ was reported for $\mathrm{Bi}_{2} \mathrm{Te}_{3},{ }^{1}$ resulting in a $Z T$ value of 0.9 at $300 \mathrm{~K}$. However, these materials are toxic, and unstable at high temperature. Only materials with good thermal stability open the field to high-temperature applications with high conversion efficiency for large temperature gradients.

Metal oxides as thermoelectric materials have been subject to extensive research in recent years due to their good chemical stability in air. ${ }^{2}$ Zinc oxide has been pointed out as a promising TE

(Received July 22, 2011; accepted November 30, 2011;

published online December 21, 2011) material $^{3}$ due to its high Seebeck coefficient, lowcost production, and nontoxicity. However, zinc oxide also exhibits high electrical resistivity and high thermal conductivity. Its electrical resistivity is improved by substitution of zinc with $2 \mathrm{~mol} \%$ aluminum. ${ }^{3}$ Substitution of $\mathrm{Al}$ in the $\mathrm{ZnO}$ lattice is a typical example of $n$-type doping in semiconductors, leading to higher electron concentration and therefore lower resistivity in the material. ${ }^{4}$ The electrical resistivity decreases without any strong influence on the Seebeck coefficient, resulting in $Z T$ of 0.3 at $1275 \mathrm{~K}$ for $2 \% \mathrm{Al}$-substituted $\mathrm{ZnO} .^{3}$ This $Z T$ value can be considered as an acceptable value for oxide materials, but much work has to be done to implement $\mathrm{ZnO}: \mathrm{Al}$ in thermoelectric devices. Its longterm stability and the effect of thermal cycling have not yet been explored. Endurance tests to study these parameters are suitable to verify the reliability of the material for applications. They are being addressed by the thermal analysis of the thermoelectric properties.

In spite of the considerable amount of research done on the thermoelectric properties of Al-substituted $\mathrm{ZnO}$, the results vary among different groups. $^{3,5-14}$ This is clearly seen in Table I, where the dispersion of data collected from several reports is 
summarized. The main reason for the experimental disagreement is based on the synthesis/sintering method (SSR, solid-state reaction; SC, soft chemistry; SPS, spark plasma sintering) and gas atmosphere during sintering and thermoelectric measurements; for example, SSR samples sintered in air show higher resistivity and higher absolute Seebeck coefficient values compared with those sintered in nitrogen, ${ }^{3,5,9}$ while samples synthesized by SC show lower absolute values for both properties than SSR samples (Table I). Other parameters such as morphology and density play an important role in the transport properties as well. They are not listed in Table I as the data are not entirely available in the literature. The behavior of the resistivity of $\mathrm{ZnO}: \mathrm{Al}$ as a function of temperature is also a subject of disagreement, since some works show metallic behavior, ${ }^{3,8}$ whereas others report semiconducting behavior ${ }^{11}$ or even show a change from semiconducting to metallic behavior depending on the $\mathrm{Al}$ content. ${ }^{8}$

A survey of the different results suggests that the transport properties of $\mathrm{Al}$-substituted $\mathrm{ZnO}$ strongly depend on the content of $\mathrm{Al}, \mathrm{Zn}$, and $\mathrm{O}$ as well as on the morphology and density of the sample. In SSR synthesis and posterior sintering, ions diffuse to form a solid solution with substitution of $\mathrm{Zn}^{2+}$ by $\mathrm{Al}^{3+}$ in the $\mathrm{ZnO}$ lattice. This is likely to occur due to the smaller ionic radius of $\mathrm{Al}^{3+}(0.049 \mathrm{~nm})$ compared with $\mathrm{Zn}^{2+}(0.071 \mathrm{~nm}) .{ }^{15}$ Shirouzu and coworkers ${ }^{16,17}$ showed substitution of $\mathrm{Al}$ ions in the $\mathrm{ZnO}$ with a change in the lattice constants by x-ray diffraction (XRD) measurements. $\mathrm{Al}$ ions need to diffuse from $\mathrm{Al}_{2} \mathrm{O}_{3}$ particles into the $\mathrm{ZnO}$, and it is reported that the expansion during sintering can only be explained by codiffusion of both $\mathrm{Zn}$ and $\mathrm{Al}$ ions in the $\mathrm{Zn}-\mathrm{Al}-\mathrm{O}$ system. ${ }^{18}$

In $\mathrm{SC}$ synthesis, $\mathrm{Al}$ and $\mathrm{Zn}$ ions are mixed in the liquid phase, and a network is formed between the ions. The homogeneous distribution of the ions is sustained in the solid phase, and a solid solution of $\mathrm{Al}$ can form in the $\mathrm{ZnO}$ lattice without diffusion of ions. $\mathrm{SC}$ synthesis is suitable for formation of low-level substituted metal oxides to obtain homogeneous distribution of the substitution element. ${ }^{19,20}$

The $\mathrm{Zn}$ and $\mathrm{O}$ contents also depend on defects in the $\mathrm{ZnO}$ lattice, which can be generated or annihilated during synthesis and sintering processes depending on the type of gas and the temperature. Generally, $\mathrm{ZnO}$ is rarely found to be stoichiometric. Heating $\mathrm{ZnO}$ to temperatures around $1200 \mathrm{~K}$ and higher leads to continuous evaporation of oxygen from particle surfaces, resulting in excess $\mathrm{Zn}$ in the lattice, which segregates on the surface and evaporates when the evaporation temperature of liquid zinc is reached. This continuous process can lead to complete dissociation of $\mathrm{ZnO}$ at temperatures above $1700 \mathrm{~K}$ as used in high-temperature solar processes. ${ }^{21}$

Variations in thermal treatment and oxygen partial pressure induce oxygen uptake or release. ${ }^{21}$ Defects formed thereby have an influence on the electron concentration and therefore the electronic properties. A decrease of carrier concentration was observed in samples sintered in increasing oxygen partial pressure. $^{22}$

In this work the thermoelectric properties of Al-substituted $\mathrm{ZnO}$ bulk samples are compared for different synthesis methods and measurement gases. $\mathrm{Zn}_{0.98} \mathrm{Al}_{0.02} \mathrm{O}$ powder was synthesized by a soft chemistry (SC) method from $\mathrm{Zn}$ and $\mathrm{Al}$ nitrates with citric acid. The properties of the pressed pellets were compared with those of $\mathrm{Zn}_{1-x} \mathrm{Al}_{x} \mathrm{O}(x=0.02$, 0.06 ) samples synthesized by solid-state reaction (SSR) processed with planetary milling. Application in thermoelectric modules requires long-term stability of the material during heating and cooling periods. Cycling during thermoelectric measurements was performed to study the aging process, phase segregation, defect formation, and changes of the thermoelectric properties.

\section{EXPERIMENTAL PROCEDURES}

Aluminum-substituted zinc oxide was prepared by soft chemistry synthesis (SC) and by solid-state

Table I. Summary of thermoelectric properties of $\mathrm{ZnO}$ :Al (2\%) depending on the synthesis/sintering method and measurement gas

\begin{tabular}{|c|c|c|c|c|c|c|}
\hline $\begin{array}{l}\text { Synthesis/ } \\
\text { Sintering }\end{array}$ & $\begin{array}{c}\text { Sintering } \\
\text { Gas }\end{array}$ & $\begin{array}{c}\text { Measurement } \\
\text { Gas }\end{array}$ & $\begin{array}{l}\text { Resistivity } \\
\quad(\Omega \text { cm) }\end{array}$ & $\begin{array}{c}\text { Seebeck } \\
(\mu \mathrm{V} / \mathrm{K})\end{array}$ & $T(\mathbf{K})$ & References \\
\hline SSR & Air & Air & $2.5 \times 10^{-3}$ & -180 & 1275 & 3 \\
\hline SSR & $\mathrm{N}_{2}$ & Air & $1 \times 10^{-3}$ & -140 & 1275 & 9 \\
\hline SSR & Air and $N_{2}$ & & $2 \times 10^{-2}$ (air) & -125 (air) & 300 & 5 \\
\hline SSR & & Inert gas $(\mathrm{Ar}, \mathrm{He})$ & $2 \times 10^{-1}$ & -180 & 1075 & 11 \\
\hline SSR + SPS & & Vacuum & 1.25 & -350 & 1275 & 6 \\
\hline & Air & Inert gas $(\mathrm{Ar}, \mathrm{He})$ & $1 \times 10^{-3}$ & -65 & 1050 & 10 \\
\hline $\mathrm{SC}+\mathrm{SPS}$ & Air & Air & $7 \times 10^{-4}$ & -60 & 1200 & 13 \\
\hline
\end{tabular}

SSR, solid-state reaction; SC, soft chemistry; SPS, spark plasma sintering. 
reaction (SSR). $\mathrm{SC} \mathrm{Zn}_{0.98} \mathrm{Al}_{0.02} \mathrm{O}$ was produced by thermal decomposition of the corresponding polymeric citrate precursors. ${ }^{23}$ The required amount of $\mathrm{Zn}\left(\mathrm{NO}_{3}\right)_{2} \cdot 6 \mathrm{H}_{2} \mathrm{O}$ (Sigma Aldrich, 98\%) and $\mathrm{Al}\left(\mathrm{NO}_{3}\right)_{3} \cdot 9 \mathrm{H}_{2} \mathrm{O}$ (Sigma-Aldrich, puriss. p.a.) was dissolved in water. Citric acid (Alfa Aesar 99+\%) was added as a chelating ligand to complex the different cations. The solution was homogenized at $373 \mathrm{~K}$ for $4 \mathrm{~h}$ under continuous stirring and reflux. The precursor solution was dried by removing the reflux to promote the formation of a colorless viscous gel. The precursor was heated up to $573 \mathrm{~K}$ at heating rate of $20 \mathrm{~K} / \mathrm{min}$ and decomposed at $573 \mathrm{~K}$ for $2 \mathrm{~h}$. The phases were obtained by grinding and calcination of the amorphous xerogel at $1073 \mathrm{~K}$.

For SSR of $\mathrm{Zn}_{1-x} \mathrm{Al}_{x} \mathrm{O} \quad(x=0.02,0.06)$, the required amount of $\mathrm{ZnO}$ (Aldrich, nanopowder $<100 \mathrm{~nm})$ and $\gamma-\mathrm{Al}_{2} \mathrm{O}_{3}$ was combined. $\left(\gamma-\mathrm{Al}_{2} \mathrm{O}_{3}\right.$ powder synthesized by the Department of Molecular and Material Sciences of Interdisciplinary Graduate School of Engineering Science at Kyushu University, group of Prof. M. Ohtaki). Two different processing routes were chosen. In the normal route the precursors were dispersed in ethanol and mixed in an agate mortar. Alternatively, the precursor powders were mixed by planetary milling with $1-\mathrm{mm}$ $\mathrm{ZrO}_{2}$ balls for $4 \mathrm{~h}$ at $300 \mathrm{rpm}$.

The polycrystalline samples from both synthesis methods were pressed into circular pellets and sintered at $1673 \mathrm{~K}$ for $10 \mathrm{~h}$ in air. Table II presents an overview of the different synthesis and processing methods, indicating the nomenclature used for the described samples.

Phase purity of the samples was confirmed by x-ray diffraction (XRD) with a PANalytical X'pert diffractometer using $\mathrm{Cu} \mathrm{K}_{\alpha}$ radiation. The morphology of the calcined powders was studied using scanning electron microscopy (SEM, Hitachi S-4800) in secondary electron (SE) and back-scattered electron (BSE) modes. The cationic composition was investigated by energy-dispersive X-ray spectroscopy (EDS) in SEM.

Sintered pellets were used to determine transport and thermal properties in air and oxygen gas under ambient pressure. The thermal conductivity $\kappa$ was calculated by the following equation: $\kappa=d \alpha C_{p}$, where $d$ is the bulk density determined by the geometrical method. The thermal diffusivity $\alpha$ was measured by the laser flash method using a Netzsch LFA-457 apparatus from $300 \mathrm{~K}$ to $1275 \mathrm{~K}$ in air. The heat capacity $C_{p}$ was measured by differential scanning calorimetry using a Netzsch DSC 404C Pegasus. The transport property measurements were done on bar-shaped pellets cut from circular pellets with general dimensions of $1.5 \mathrm{~mm} \times$ $1.5 \mathrm{~mm} \times 8.0 \mathrm{~mm}$. The electrical conductivity and Seebeck coefficient were measured in air and in oxygen as a function of temperature from $313 \mathrm{~K}$ to $1275 \mathrm{~K}$ using a RZ2001i measurement system from Ozawa Science, Japan. The electrical conductivity was determined using the four-point probe method. Two electrical contacts were positioned at both ends of the sample, and the two other contacts were on the sample body. The contacts at both ends were fixed mechanically with Pt layers. The contacts on the sample were made by surrounding the barshaped sample with Pt wires.

\section{RESULTS AND DISCUSSION}

\section{Characterization}

The x-ray powder diffraction pattern from SC-synthesized $\mathrm{Zn}_{0.98} \mathrm{Al}_{0.02} \mathrm{O}$ in Fig. 1 shows the wurtzite structure with no secondary phase. Figure 1 also compares the XRD patterns of fresh samples as synthesized by $\mathrm{SC}$ with the pattern of sintered pellets before and after polishing (SC2NR). All reflections are normalized to the most intense one (101). After sintering at $1673 \mathrm{~K}$ the $\mathrm{ZnAl}_{2} \mathrm{O}_{4}$ spinel phase appears. As there is no Al-phase observed in the as-prepared powder, the $\mathrm{Al}$ for the $\mathrm{ZnAl}_{2} \mathrm{O}_{4}$ formed during sintering has to result from $\mathrm{Al}$ diffusion out of the $\mathrm{ZnO}: \mathrm{Al}$ phase. Coexistence of this secondary phase together with $\mathrm{Zn}_{1-x} \mathrm{Al}_{x} \mathrm{O}$ has been reported by several authors. ${ }^{3,5,6,13,15}$ To avoid the formation of the spinel phase, the samples were introduced into the furnace at $1275 \mathrm{~K}$ and quenched from that temperature. Kim et al. ${ }^{6}$ reported the formation temperature of $\mathrm{ZnAl}_{2} \mathrm{O}_{4}$ to be between $1073 \mathrm{~K}$ and $1273 \mathrm{~K}$. Despite avoiding this temperature, a considerable amount of the secondary phase was formed, showing high stability of the spinel

Table II. Overview and nomenclature of samples from different synthesis and processing methods

\begin{tabular}{lccccc}
\hline Synthesis & $\begin{array}{c}\text { Al } \\
\text { Substitution (\%) }\end{array}$ & \multicolumn{2}{c}{ Normal Route } & & $\begin{array}{c}\text { Planetary } \\
\text { Milling }\end{array}$ \\
& 2 & & SC2NR & - \\
SC & 2 & & SC2NR-air & SC2NR-O2 & SSR2PM \\
SSR & 6 & & SSR2NR & & SSR6PM \\
SSR & & & - & \\
\hline
\end{tabular}

SC, soft chemistry; SSR, solid-state reaction; air, measurement in air; O2, measurement in oxygen; if not mentioned, measurement was in air. 


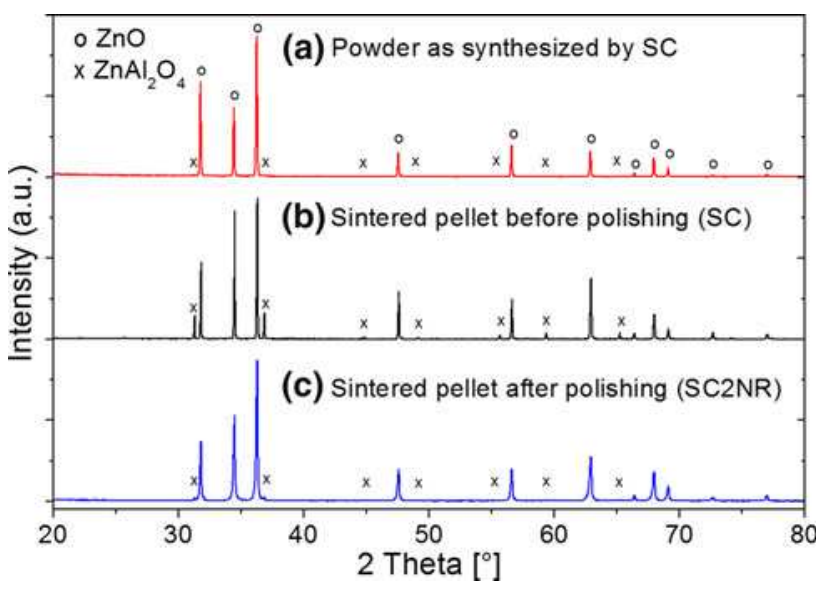

Fig. 1. X-ray diffraction patterns of SC samples with $2 \%$ Al substitution: (a) calcined powder, (b) sintered pellet before polishing, and (c) sintered pellet after polishing.

phase $\mathrm{ZnAl}_{2} \mathrm{O}_{4}$ over a broad temperature range. Information on the stability of the spinel phase in the $\mathrm{Zn}-\mathrm{Al}-\mathrm{O}$ system is rare and incomplete. The largest amount of the spinel phase was found at the surface of the sintered samples, as also reported in Ref. 5. After polishing, the reflection intensity of the secondary phase decreased to a small amount (Figs. 1, 2), thus the method of polishing to remove the spinel phase is efficient enough.

Figure 2 compares XRD patterns of sintered SC (SC2NR) and SSR samples (SSR2NR, SSR2PM, and SSR6PM) in pellet shape after polishing. The amount of $\mathrm{ZnAl}_{2} \mathrm{O}_{4}$ after polishing differs between the different synthesis processes, treatments, and compositions. However, all samples show coexistence of both $\mathrm{ZnO}$ and $\mathrm{ZnAl}_{2} \mathrm{O}_{4}$ structures. The intensity of the secondary-phase reflections seems to be highest for 6\% $\mathrm{Al}$ substitution (SSR6PM). In SSR samples with $2 \% \mathrm{Al}$ substitution, there is less secondary phase observed for the sample with planetary milling treatment (SSR2PM) than for the normal route with mortar mixing (SSR2NR). The amount of $\mathrm{ZnAl}_{2} \mathrm{O}_{4}$ phase formed is too small to quantify the difference between the processing methods using XRD, especially between the SSR and SC synthesis methods.

Figure 3 shows SEM images of the middle body of sintered samples from different synthesis processes. Relative density of around 95\% was reached for all samples except SSR2NR, which was less than $90 \%$ dense. The SEM images reveal differences in the size and amount of pores. Planetary milling of SSR powder led to fewer and smaller pores compared with the normal route. There are also fewer and smaller pores in samples prepared by SC than by SSR with the normal route. For samples SSR6PM and SSR2NR, spinel phase $\mathrm{ZnAl}_{2} \mathrm{O}_{4}$ particles can be observed. Areas showing these particles are marked with frames in Fig. 3. Moreover, after sintering, a considerable amount of spinel phase can be

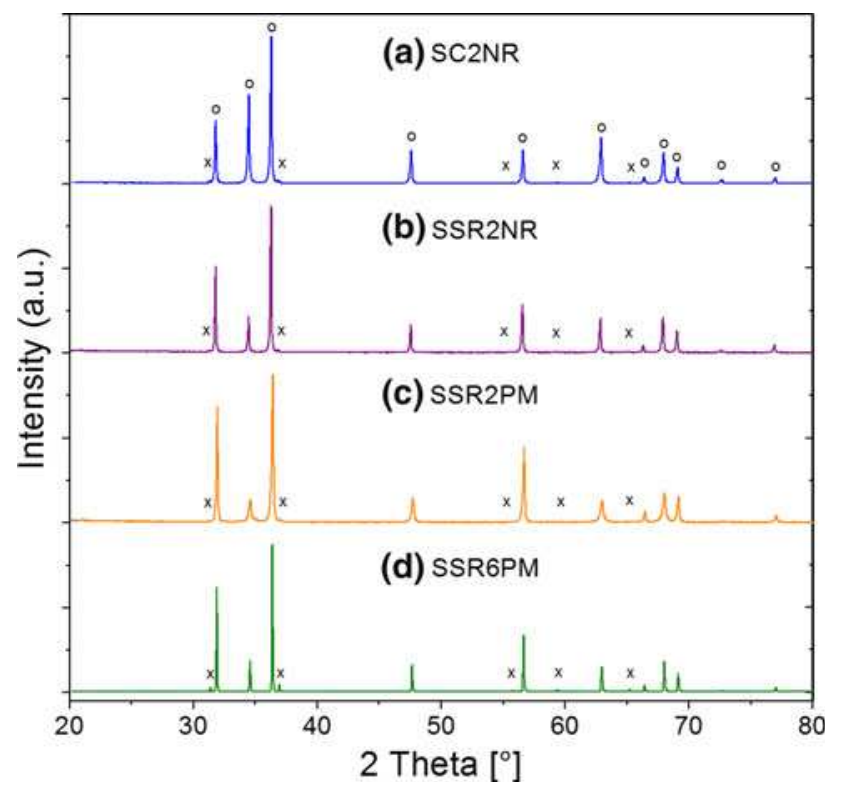

Fig. 2. X-ray diffraction patterns of sintered samples from different synthesis processes: (a) SC $2 \%$, (b) SSR $2 \%$ normal route, (c) SSR $2 \%$ planetary milling, and (d) SSR $6 \%$ planetary milling.

observed on the surface, which is removed after polishing (see Supplementary Fig. S1).

The reason for the formation of $\mathrm{ZnAl}_{2} \mathrm{O}_{4}$ phase is the low solubility limit of $\mathrm{Al}$ in $\mathrm{ZnO}$, which can be as low as 0.3 at.\% depending on the synthesis method. ${ }^{15}$ The excess $\mathrm{Al}$ reacts with $\mathrm{ZnO}$ to form the spinel phase $\mathrm{ZnAl}_{2} \mathrm{O}_{4}$. A greater amount of added $\mathrm{Al}$ leads to a larger amount of spinel phase, as can be seen in Fig. 2 for $2 \%$ and $6 \%$ Al substitution. Planetary milling with SSR synthesis helps to decrease the particle size and enables better mixing and reaction between $\mathrm{Al}_{2} \mathrm{O}_{3}$ and $\mathrm{ZnO}$, resulting in formation of less $\mathrm{ZnAl}_{2} \mathrm{O}_{4}$ phase, as shown in Fig. 2. On the other hand, it is well known that milling balls might also be a source of low levels of impurities. The Al concentration was described to be higher at grain boundaries and at the sample surface due to segregation. ${ }^{16}$ This segregation and the fact that large space is needed by the spinel phase during formation due to lattice expansion ${ }^{18}$ could be the reasons for the formation of a large amount of $\mathrm{ZnAl}_{2} \mathrm{O}_{4}$ phase at the surface during the sintering process, as observed by XRD (Fig. 1) and SEM (SE/ BSE) (Supplementary Fig. S1).

\section{Thermoelectric Properties}

Figure 4 shows the resistivity and Seebeck coefficient values as a function of temperature for sample SC2NR-air in four cycles measured in air. The cycling leads to aging of the sample with a change in the transport properties. The electrical resistivity increases continuously by about $25 \%$ over all four cycles. The increase of the absolute value of the Seebeck coefficient is about $10 \%$. The source of the errors shown for all the measurement variables 

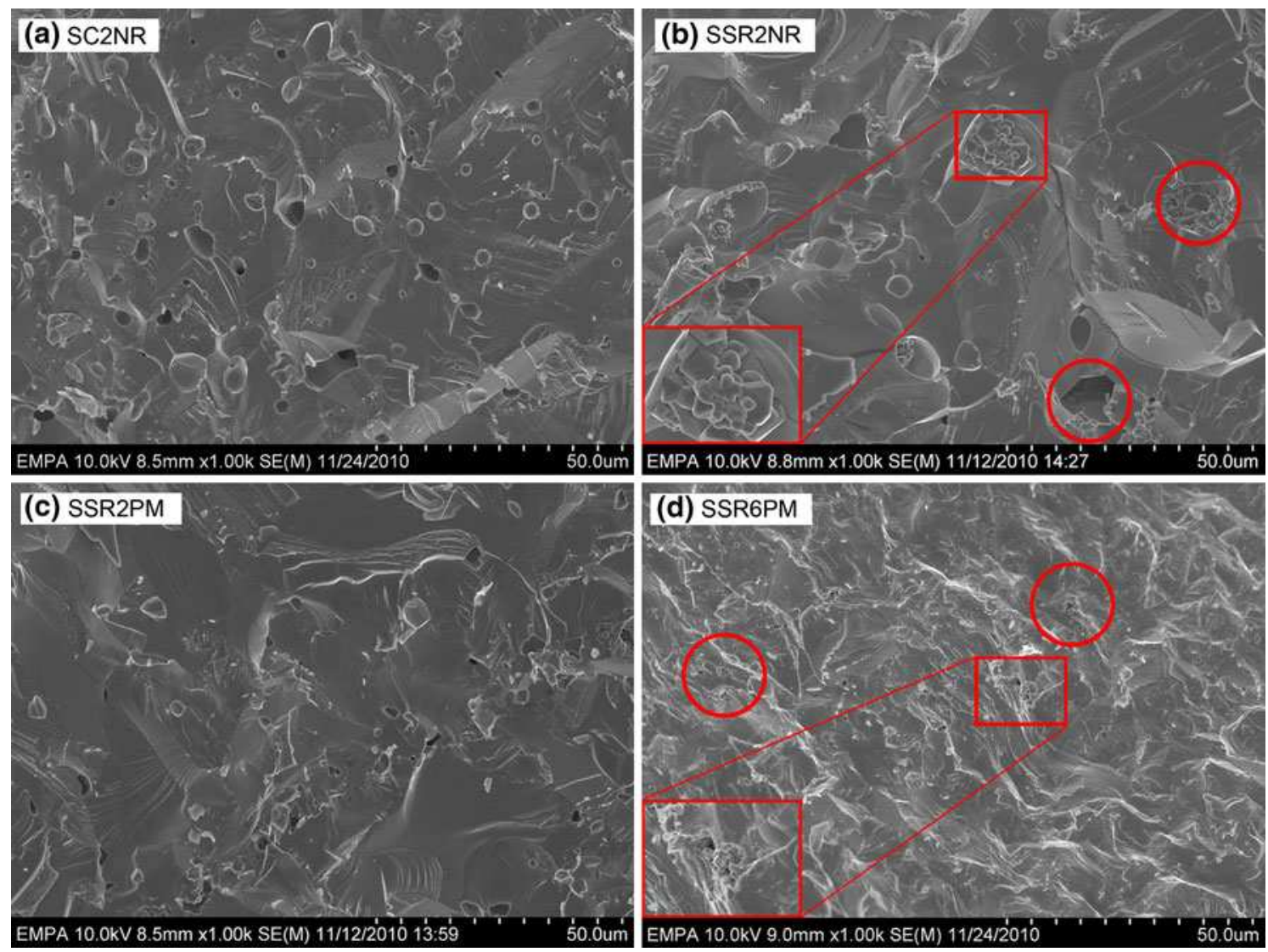

Fig. 3. SEM images of sintered samples from different synthesis processes: (a) SC2NR, (b) SSR2NR, (c) SSR2PM, and (d) SSR6PM; frames mark areas with spinel phase $\mathrm{ZnAl}_{2} \mathrm{O}_{4}$ particles.

is described in detail in the Supplementary Information. The evolution of the transport properties can be explained by a decrease of the carrier concentration leading to an increase in resistivity and absolute Seebeck coefficient values. ${ }^{3}$ This change in the charge carrier concentration can result from oxygen uptake by the material during measurement in air atmosphere, leading to a decreased defect concentration. Another influence which has to be considered in the aging of the sample during cycling is diffusion of $\mathrm{Al}$ out of grains, leading to lower $\mathrm{Al}$ substitution. Slight excess of $\mathrm{Al}$ integrated into $\mathrm{ZnAl}_{2} \mathrm{O}_{4}$ particles on the surface of sintered pellets of SC sample SC2NR was detected by XRD (Fig. 1). In the XRD pattern of as-synthesized SC powder, no $\mathrm{ZnAl}_{2} \mathrm{O}_{4}$ phase could be observed. The source of $\mathrm{Al}$ to form the spinel phase on the surface during sintering has to originate from $\mathrm{Al}$ diffusing out of $\mathrm{Zn}_{1-x} \mathrm{Al}_{x} \mathrm{O}$ grains. The segregation of $\mathrm{Al}$ at the surface and in grain boundaries enhances the formation of $\mathrm{ZnAl}_{2} \mathrm{O}_{4}$ due to its greater stability. The small amount of $\mathrm{ZnAl}_{2} \mathrm{O}_{4}$ in the $\mathrm{ZnO}: \mathrm{Al}$ sample observed in this work is not supposed to have an influence on the thermoelectric properties, ${ }^{12}$ as the electrical resistivity is high, being $10^{6} \Omega \mathrm{cm}$ at $1073 \mathrm{~K}^{24}$ Due to this high resistivity, the Seebeck coefficient could not be measured. Comparison of the XRD or SEM/EDX results for the samples before versus after the thermoelectric property measurements did not show a detectable difference in the amount of $\mathrm{ZnAl}_{2} \mathrm{O}_{4}$.

The first heating curve differs significantly from the rest of the curves, especially in showing a lower absolute value of the Seebeck coefficient. This effect is pronounced in the resistivity and Seebeck coefficient measurement of sample SSR2NR (Fig. 5). Both values are much lower for the first heating cycle than for the subsequent cooling. This effect can be explained because the samples were quenched quickly after sintering from $1275 \mathrm{~K}$ to room temperature. This causes a freezing of the hightemperature phase with defects such as oxygen vacancies or zinc interstitials from oxygen desorption. ${ }^{21,22}$ Loss of oxygen at high temperature leads to nonstoichiometric $\mathrm{Zn}_{1+x} \mathrm{O}$ with $x=0.00007$ at $1075 \mathrm{~K}^{25}$ The obtained samples glow yellow at $1275 \mathrm{~K}$, and a yellowish color can be observed for the quenched samples at room temperature. It is described that excess zinc atoms lead to a yellow color of $\mathrm{ZnO}^{25}$ The nonstoichiometric defects are maintained during the fast cooling after sintering. The higher carrier concentration due to the defects leads to low resistivity and Seebeck coefficient in the first heating cycle. At around $1075 \mathrm{~K}$ the electrical resistivity and the absolute value of the Seebeck coefficient increase, which can be explained by a 


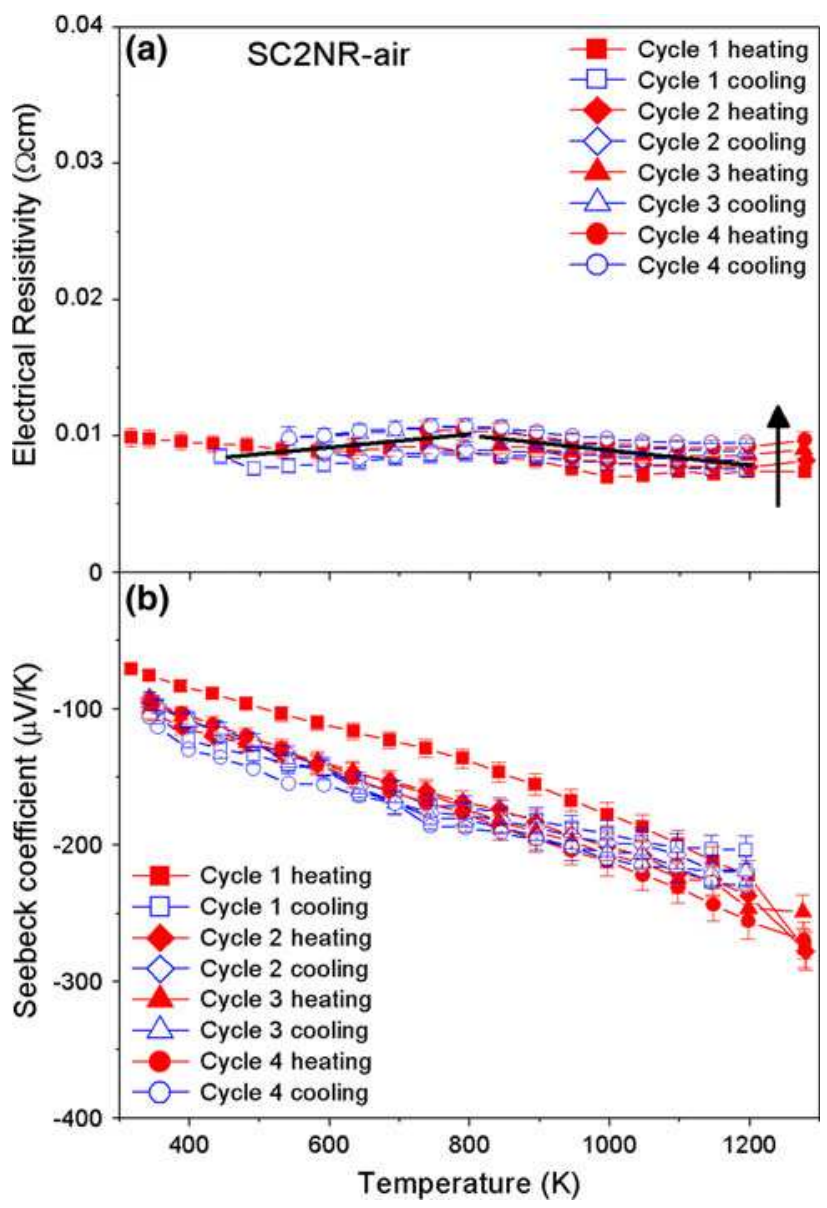

Fig. 4. (a) Electrical resistivity and (b) Seebeck coefficient as a function of temperature for sample SC2NR-air; lines are a guide to the eye, showing the change from metallic to semiconductor behavior (errors: resistivity $6.5 \%$, Seebeck coefficient $5 \%$ ).

minimization of oxygen vacancies due to oxygen uptake. The thermal stability of $\mathrm{ZnO}$ in flowing synthetic air was tested in thermal analysis experiments. Weight loss of $0.3 \mathrm{wt} . \%$ was observed between room temperature and $500 \mathrm{~K}$, resulting from desorption of $\mathrm{CO}_{2}$ and water from the surface. Up to $1675 \mathrm{~K}$ no significant weight changes were detected, indicating that the change in stoichiometry lies in a range not observed in the thermal analysis experiments. At high temperature, the experimental values from different cycles converge. However, at $1275 \mathrm{~K}$ the resistivity values of SSR2NR increase with cycling and show a similar aging process to SC2NR-air. The difference between the first heating and the first cooling cycle is much more noticeable in SSR2NR (Fig. 5) than in SC2NRair (Fig. 4). The reason for this is the lower density of around $90 \%$ for SSR2NR, compared with SC2NRair that reached a density of around $95 \%$. The lower density, due to the existence of pores, favors oxygen loss and uptake. The SEM images in Fig. 3 show larger pores of $5 \mu \mathrm{m}$ to $30 \mu \mathrm{m}$ for SSR2NR compared with pores smaller than $10 \mu \mathrm{m}$ for SC2NR-air. The lower density is also the origin of the slightly higher

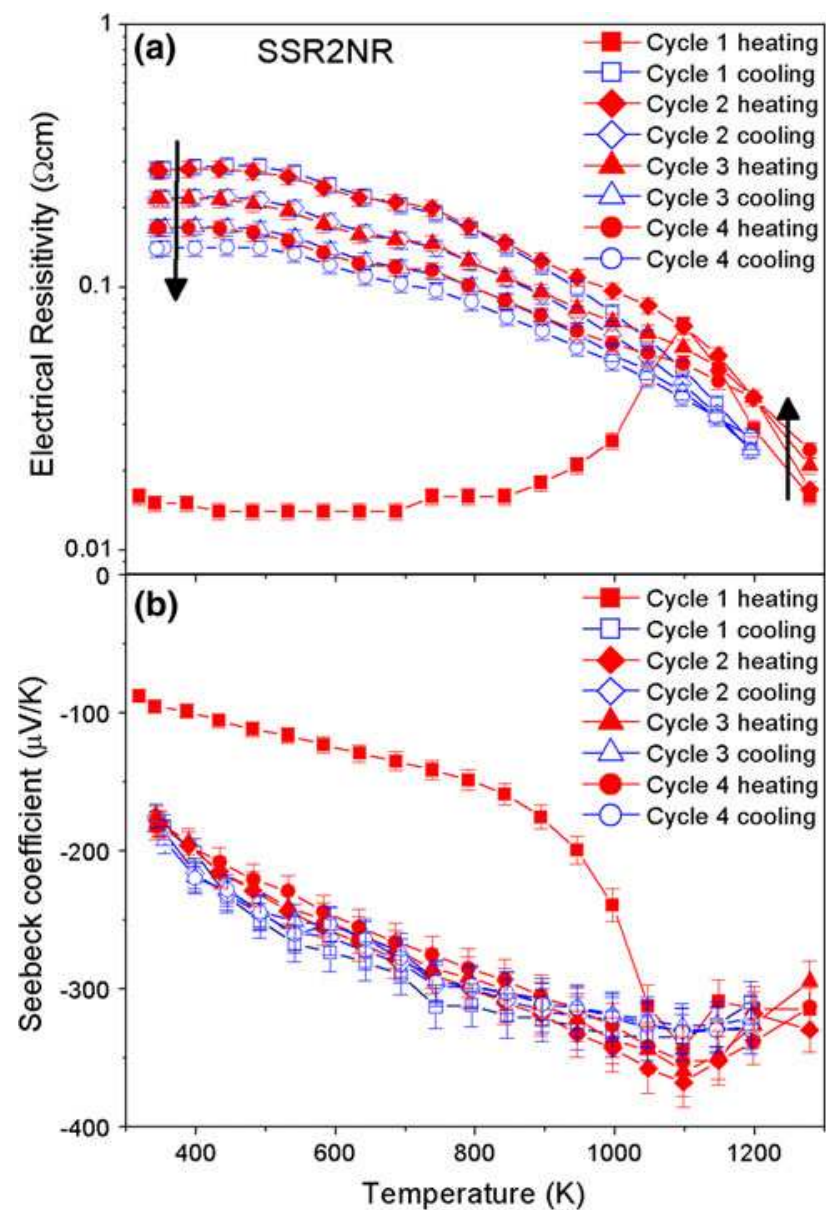

Fig. 5. (a) Electrical resistivity and (b) Seebeck coefficient as a function of temperature for sample SSR2NR (errors: resistivity $7 \%$, Seebeck coefficient $5 \%$ ).

resistivity of SSR2NR. The decrease of the resistivity in the second cycle at low temperature (Fig. 5) can be explained by an enhancement of the connectivity between grains with a negligible effect of compositional change. This is confirmed by the observation of the Seebeck coefficient, which changes by less than $5 \%$ in four cycles. The density is an important factor for the thermoelectric properties. Our studies on the influence of the density revealed that data reproduction was difficult in the case of densities as low as $60 \%$. However, the improvement of the sintering process with an increase of temperature and sintering time reaching the described values ensured reproducible results.

Preparing the SSR sample by planetary milling (SSR2PM) instead of the preceding route (SSR2NR) produced a higher density of sintered pellets with less change in the microstructure during different cycles in the transport property measurements. The results are shown in Fig. 6. They are comparable to the results from SC2NR-air (Fig. 4) for the aging process with an increase of the resistivity by $20 \%$ and of the absolute value of the Seebeck coefficient by $10 \%$. However, the resistivity values are twice as 


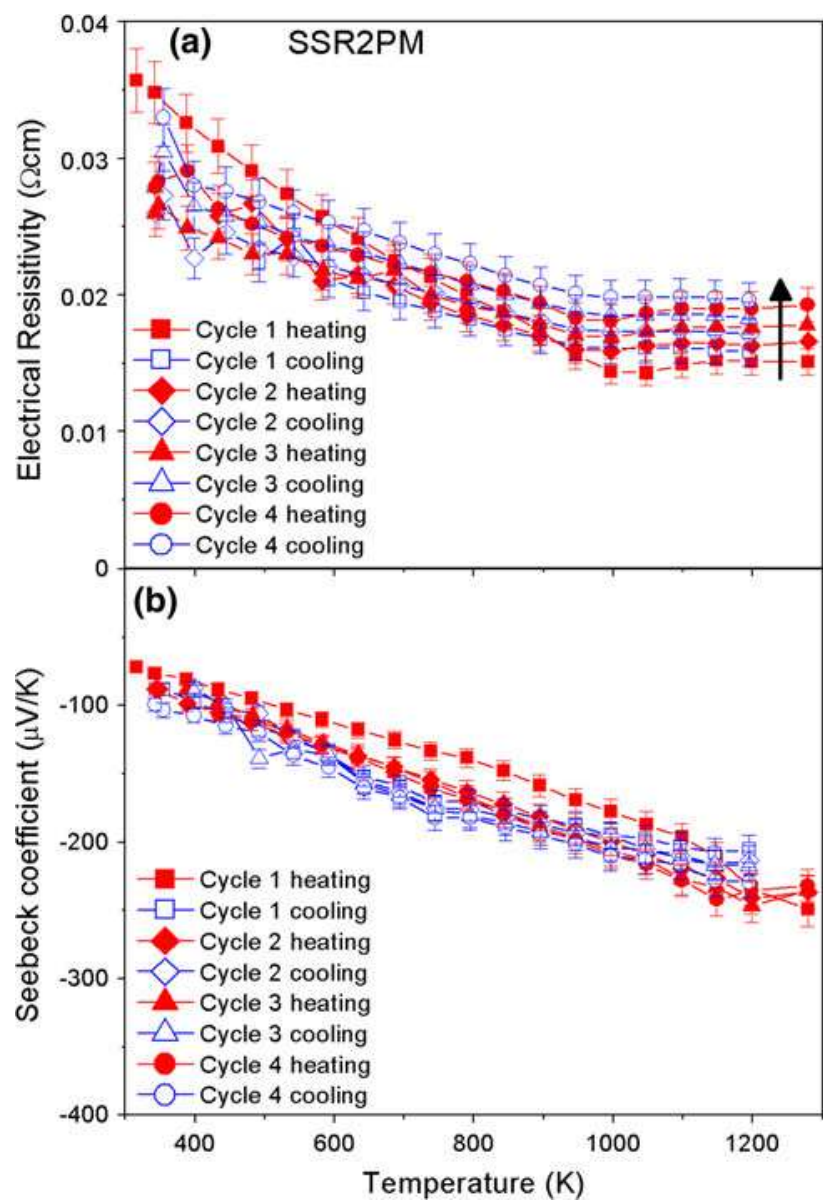

Fig. 6. (a) Electrical resistivity and (b) Seebeck coefficient as a function of temperature for sample SSR2PM (errors: resistivity $6.5 \%$, Seebeck coefficient 5\%).

high for SSR2PM as for SC2NR-air at high temperature, and four times as high at low temperature. This difference in electrical resistivity could be a result of better connectivity between grains in SC2NR-air compared with SSR2PM, since the densities of the two samples are in the same range of about $95 \%$ and the Seebeck coefficient values are similar in both cases. Nevertheless, EDX analysis in SEM revealed a minor impurity which was not detectable in XRD and that could have been introduced by the ball milling process due to abrasion. This is another possible explanation for the increase of the resistivity by $0.01 \Omega \mathrm{cm}$ compared with sample SC2NR-air. Study of the grain connectivity comparing the different synthesis methods is in progress.

For SC2NR-air, the resistivity changed from metallic to semiconducting behavior with temperature, while SSR2PM showed semiconducting behavior in this work.

Sample SSR6PM containing 6\% Al exhibited absolute values of the Seebeck coefficient about $50 \mu \mathrm{V} / \mathrm{K}$ lower than SSR2PM with $2 \% \mathrm{Al}$ substitution. The results for resistivity, Seebeck coefficient, and power factor for 6\% $\mathrm{ZnO}$ :Al (SSR6PM) compared with $2 \% \mathrm{ZnO}: \mathrm{Al}$ (SC2NR and SSR2PM) are shown in Supplementary Fig. S2. The difference in Seebeck coefficient can be attributed to a greater amount of $\mathrm{Al}$ in the $\mathrm{ZnO}$ lattice due to the higher $\mathrm{Al}$ substitution. Substitution of $\mathrm{Zn}^{2+}$ by $\mathrm{Al}^{3+}$ ions produces higher electron concentration ${ }^{7}$ and should therefore decrease the electrical resistivity, which is corroborated in samples SSR6PM and SSR2PM with $6 \%$ and $2 \% \mathrm{Al}$ substitution, respectively, in Supplementary Fig. S2. The best power factor of $1 \mathrm{~mW} / \mathrm{K}^{2} \mathrm{~m}$ was found at $1273 \mathrm{~K}$ in sample SC2NRair, followed by SSR6PM.

The measurement gas is another important parameter that influences the transport properties of Al-substituted $\mathrm{ZnO}$. The role of oxygen in the transport properties was checked in heating and cooling cycles in oxygen gas (SC2NR-O2). Figure 7 shows a comparison of the electrical resistivity and Seebeck coefficient values of SC2NR-O2 and SC2NR-air. The first cycle is the same in both measurement gases regarding the resistivity and the Seebeck coefficient. However, the aging effect with increasing number of cycles is more pronounced in oxygen than in air. The resistivity increases by $60 \%$ in oxygen compared with $25 \%$ in air. The change in the absolute value of the Seebeck coefficient is also larger in oxygen with a rise of $15 \%$. This is because more oxygen can be absorbed by the material when performing the measurements in flowing oxygen gas. This reduces the available charge carriers and increases the resistivity and the absolute value of the Seebeck coefficient.

Figure 8 shows the thermal conductivity values of the samples made by different synthesis processes as a function of temperature. SSR2PM shows lower thermal conductivity values than SC2NR-air. This can be explained by insufficient connectivity of the grains in SSR2PM or the influence of scattering at impurities. Evidence for this is provided by the smaller fractional electrical contribution to the thermal conductivity $\left(\kappa_{\mathrm{el}}\right)$ for SSR2PM compared with SC2NR-air. The total thermal conductivity $\left(\kappa_{\mathrm{T}}\right)$ is composed of $\kappa_{\mathrm{el}}$ and the lattice $\left(\kappa_{\mathrm{ph}}\right)$ thermal conductivity as $\kappa_{\mathrm{T}}=\kappa_{\mathrm{el}}+\kappa_{\mathrm{ph}} \cdot \kappa_{\mathrm{el}}$ is connected to the electrical conductivity $\sigma$ by the Wiedemann-Franz relation $\kappa_{\mathrm{el}}=L \sigma T$ (where $L$ is the Lorenz number and $T$ is absolute temperature). ${ }^{3}$ The lower fractional contribution of the electron thermal conductivity in SSR2PM means that the part of heat conducted by electrons is more inhibited than in SC2NR-air, for example, due to poor connectivity between grains or electron scattering by impurities introduced by ball milling. The total thermal conductivities for SC2NR-air and SSR6PM are similar at intermediate and high temperatures, even though the percentage contribution of $\kappa_{\mathrm{el}}$ is higher for SSR6PM than for SC2NR-air. This means that $\kappa_{\mathrm{ph}}$ for SSR6PM should have lower values than for SC2NR-air. A possible reason is the greater amount of small spinel phase $\mathrm{ZnAl}_{2} \mathrm{O}_{4}$ particles in SSR6PM, which can act as scattering centers for phonons, lowering the thermal conductivity. ${ }^{12}$ 


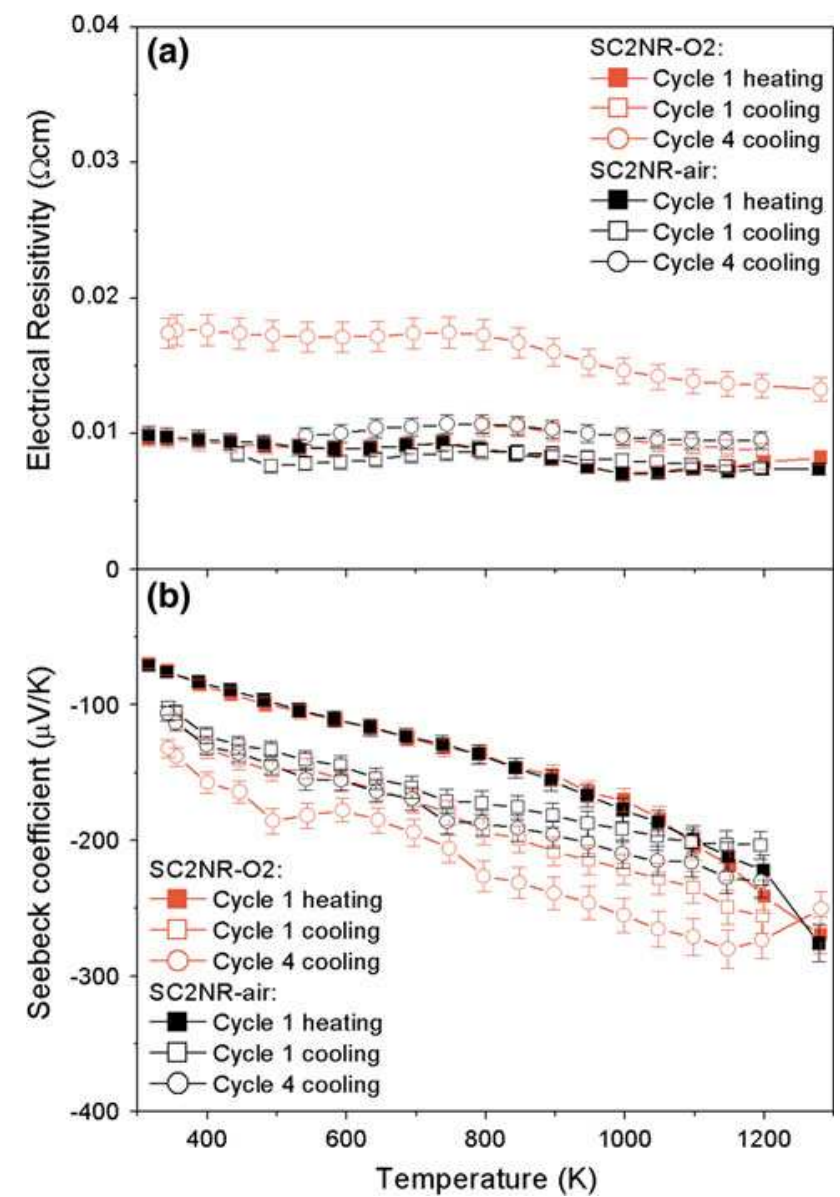

Fig. 7. Comparison of (a) electrical resistivity and (b) Seebeck coefficient as a function of temperature for samples SC2NR-O2 and SC2NR-air (errors for both: resistivity $6.5 \%$, Seebeck coefficient $5 \%$ ).

The thermal conductivity of $\mathrm{Al}$-substituted $\mathrm{ZnO}$ hardly decreased to values lower than $5 \mathrm{~W} / \mathrm{mK}$ in all Refs. 3, 6, 7, 9, 10, 12, and 13 . The simple wurtzite crystal structure and the presence of light elements result in high thermal conduction due to lattice vibrations. ${ }^{3}$

The highest $Z T$ value of 0.21 was reached in SC2NR-air at $1275 \mathrm{~K}$. Figure 9 shows the $Z T$ values of samples made by different synthesis processes as a function of temperature. The calculation of the $Z T$ values was based on the first cooling curve for the electrical resistivity and Seebeck coefficient for all samples. Soft chemistry synthesis in this work produced a $Z T$ value at $1275 \mathrm{~K}$ that is almost an order of magnitude higher than that described by Katsuyama et al. ${ }^{13}$ for a similar soft chemistry method with spark plasma sintering. In those works lower electrical resistivity is reached, but this is counteracted by a reduced Seebeck coefficient. The $Z T$ of the SSR sample (SSR2PM) was 0.13 . The $Z T$ value decreases with increasing number of cycles, with greater stability for SC compared with SSR samples.

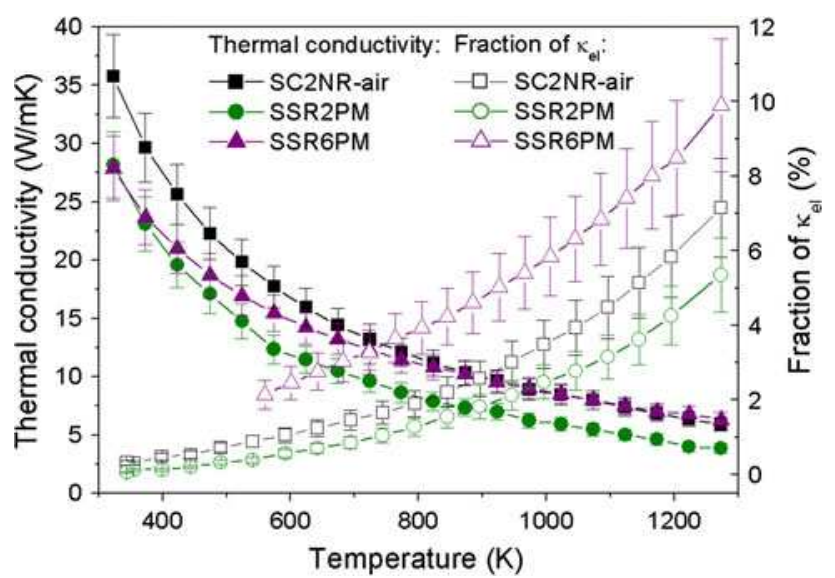

Fig. 8. Comparison of the thermal conductivity $(\kappa)$ and the fractional electrical contribution to the thermal conductivity (fraction of $\kappa_{\mathrm{el}}$ ) as a function of temperature for samples made by different synthesis processes: SC2NR-air, SSR2PM, and SSR6PM (errors: thermal conductivity $10 \%$ for all samples; fraction of $\kappa_{\mathrm{el}}$ : SC2NR-air and SSR2PM $18.5 \%$, SSR6PM 18\%).

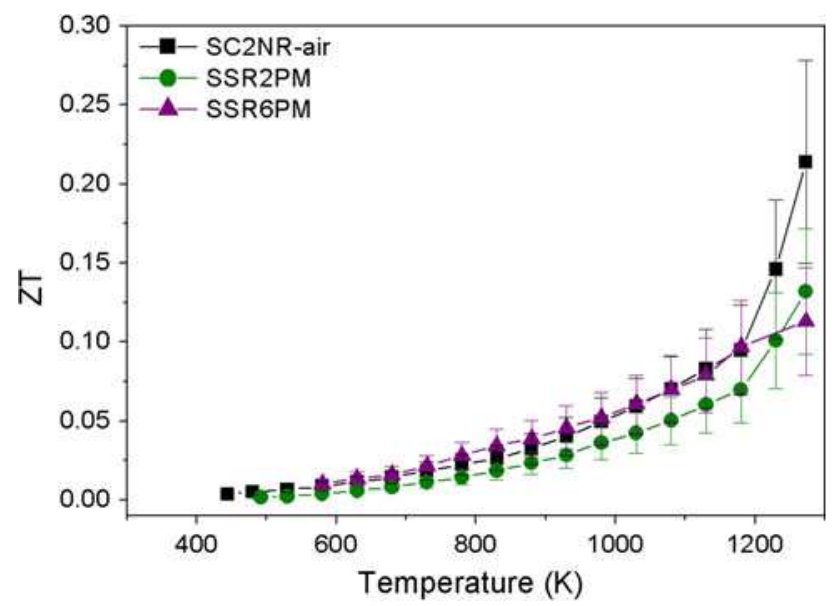

Fig. 9. Comparison of $Z T$ values as a function of temperature for samples made by different synthesis processes: SC2NR-air, SSR2PM, and SSR6PM (error: ZT 30\% for all samples).

\section{CONCLUSIONS}

Samples prepared by soft chemistry (SC) synthesis show better performance in terms of thermoelectric properties with $Z T$ of 0.21 compared with $Z T$ of 0.13 for samples prepared by solid-state reaction (SSR) for $2 \%$ Al substitution, where a minor amount of impurities due to ball milling could be found. The stability of samples prepared by SC is better than those made by SSR in long-term tests and several heating and cooling cycles.

Measurement of thermoelectric properties during temperature cycling of the samples reveals an aging process with a clear change of the transport properties. Heating-cooling cycles lead to an increase of the electrical resistivity and the absolute value of the Seebeck coefficient and a decrease of the $Z T$ value. Possible reasons for the aging process are 
oxygen absorption and $\mathrm{Al}$ diffusion out of the $\mathrm{ZnO}$ lattice, both effects producing lower carrier concentration. The variation in the transport properties is stronger in oxygen than in air atmosphere. The first heating cycle showed lower resistivity and absolute Seebeck coefficient values than the subsequent cooling, especially in samples with low density. SSR samples substituted with 6\% $\mathrm{Al}$ showed lower resistivity and absolute Seebeck coefficient values than samples with $2 \% \mathrm{Al}$ substitution. This suggests that more $\mathrm{Al}$ is substituted in the $\mathrm{ZnO}$ lattice when a greater amount of $\mathrm{Al}_{2} \mathrm{O}_{3}$ is added.

\section{ACKNOWLEDGEMENTS}

The authors thank the Swiss National Science Foundation (SNSF) for the financial support (Project No. 124688). Many thanks are given to Prof. M. Ohtaki for providing the $\gamma-\mathrm{Al}_{2} \mathrm{O}_{3}$ powder and for many fruitful discussions.

\section{ELECTRONIC SUPPLEMENTARY MATERIAL}

The online version of this article (doi:10.1007/ s11664-011-1851-2) contains supplementary material, which is available to authorized users.

\section{REFERENCES}

1. H. Scherrer, Thermoelectrics Handbook, ed. D.M. Rowe (CRC Press, 2006), p. 27-14.

2. K. Koumoto, Thermoelectrics Handbook, ed. D.M. Rowe (CRC Press, 2006), p. 35-1.

3. T. Tsubota, M. Ohtaki, K. Eguchi, and H. Arai, J. Mater. Chem. 7, 1 (1997).

4. G. Heiland and E. Mollwo, Solid State Phys. 8, 191 (1959).
5. D. Bérardan, C. Byl, and N. Dragoe, J. Am. Ceram. Soc. 93, 2352 (2010).

6. K.H. Kim, S.H. Shim, K.B. Shim, K. Niihara, and J. Hojo, J. Am. Ceram. Soc. 88, 628 (2005).

7. N. Ma, J.-F. Li, B.P. Zhang, Y.H. Lin, L.R. Ren, and G.F. Chen, J. Phys. Chem. Solids 71, 1344 (2010).

8. E. Guilmeau, A. Maignan, and C. Martin, J. Electron. Mater. 38, 1104 (2009).

9. M. Ohtaki, K. Araki, and K. Yamamoto, J. Electron. Mater. 38, 1234 (2009)

10. H. Cheng, X.J. Xu, H.H. Hng, and J. Ma, Ceram. Int. 35, 3067 (2009).

11. K. Park, K.Y. Ko, W.-S. Seo, W.-S. Cho, J.-G. Kim, and J.Y. Kim, J. Eur. Ceram. Soc. 27, 813 (2007).

12. K.F. Cai, E. Müller, C. Drasar, and A. Mrotzek, Mater. Sci. Eng. B 104, 45 (2003).

13. S. Katsuyama, Y. Takagi, M. Ito, K. Majima, H. Nagai, H. Sakai, K. Yoshimura, and K. Kosuge, J. Appl. Phys. 92, 1391 (2002).

14. Y. Tanaka, T. Ifuku, K. Tsuchida, and A. Kato, J. Mater. Sci. Lett. 16, 155 (1997).

15. K. Shirouzu, T. Ohkusa, M. Hotta, N. Enomoto, and J. Hojo, J. Ceram. Soc. Jpn. 115, 254 (2007).

16. K. Shirouzu, T. Kawamoto, N. Enomoto, and J. Hojo, Jpn. J. Appl. Phys. 49, 010201 (2010).

17. J.P. Wiff, Y. Kinemuchi, H. Kaga, C. Ito, and K. Watari, J. Eur. Ceram. Soc. 29, 1413 (2009).

18. W.-S. Hong and L.C. De Jonghe, J. Am. Ceram. Soc. 78, 3217 (1995).

19. A. Weidenkaff, Adv. Eng. Mater. 6, 709 (2004).

20. L. Bocher, M.H. Aguirre, D. Logvinovich, A. Shkabko, R. Robert, M. Trottmann, and A. Weidenkaff, Inorg. Chem. 47, 8077 (2008).

21. A. Weidenkaff, A.W. Reller, A. Wokaun, and A. Steinfeld, Thermochim. Acta 359, 69 (2000).

22. Y. Kinemuchi, C. Ito, H. Kaga, T. Aoki, and K. Watari, J. Mater. Res. 22, 1942 (2007).

23. R. Robert, S. Romer, A. Reller, and A. Weidenkaff, Adv. Eng. Mater. 7, 303 (2005).

24. A. Petric and H. Ling, J. Am. Ceram. Soc. 90, 1515 (2007).

25. A.F. Holleman, E. Wiberg, and N. Wiberg, Lehrbuch der Anorganischen Chemie, 102nd ed. (Berlin: Walter de Gruyter, 2007), p. 1492. 